\title{
Nanoherbals in Human Healthcare: A Proposed Research and Development Roadmap II
}

\author{
S. AHMAD* AND U. HASHIM \\ Institute of Nano Electronic Engineering (INEE), \\ University of Malaysia Perlis (UniMAP), Kangar, Perlis, Malaysia
}

(Abstract and key words are found in page 55 of this issue)

\section{Cosmetics, Cosmeceuticals and Nutricosmetics}

It is useful to clarify terminological differences that are frequently encountered. For example, cosmetics enhance the appearance or odor of the human body and consist of skin-care creams, lotions, powders, perfumes, lipsticks, finger/toe nail polish, eye/facial makeup, permanent waves, colored contact lenses, hair colors, sprays and gels; deodorants, baby products, bath oils, bubble baths, bath salts, butters and many other types of products. Cosmeceuticals are combinations of cosmetics plus pharmaceuticals such as anti-aging creams and moisturizers with bioactive ingredients having drug-like properties. On the other hand, nutricosmetics are the nutritional supplements which support the function and structure of the skin. The difference between the two lies in the delivery of cosmecuticals and nutricosmetics. Cosmeceuticals such as creams, lotions, and ointments are for external use whereas nutricosmetics are administered orally.

A fairly large number of plant derived compounds are currently being used in modern cosmetics industries. Most of these useful compounds have been highlighted by Mufti and Macchio (2009). Some typical phytochemicals are included here to highlight the importance of species in modern cosmetics.
A polysaccharide from oats is shown to be active in collagen production, fibroblast stimulation and protection against UVA/UVB damage besides improving the tensile strength of hair and significantly reduced breakage due to bleaching (Mufti \& Macchio 2009; Somerset Cosmetic Company). Another plant-derived carbohydrate from konjac plant containing mannose and glucose - is an extensin of a plant-derived glycoprotein similar to animalderived collagen and is used as a genuine replacement.

A new lip therapy based on palmitoyl oligopeptide showed excellent moisturizing properties. Pectin derived from carbohydrates backbone with monosaccharide side chains commonly found in vegetables and fruits, especially citrus fruit and apples; form a low residue gel useful in coloured cosmetics because of its compatibility with the pigments used (Mufti \& Macchio 2009).

An important group of compounds like saponins, flavanols and tannins are extensively used in cosmetics. Saponins are water-soluble amorphous colloidal glycosides derived from licorice and yucca that produce froth in stirred solution are widely used as natural emulsifiers in cosmetics (Mufti \& Macchio 2009; Somerset Cosmetic Company). Licorice extract is used

* Corresponding author (e-mail: drsahmad@email.com) 
as skin whitener and powerful antioxidant comparable to the enzyme dismutase, found in some vegetables, mushrooms, soy beans and spinach. Potassium and zinc salts of glycyrrhetinic acid are used as anti-inflammatory substance causing soothing action in sensitive skins (Mufti \& Macchio 2009). Tannins are natural plant polyphenols extracted from the bark of chestnut, nutgall, oak, sumac leaves, hemlock, coffee, tea and walnuts and are used as metal ion chelator, a protein precipitant and a biological antioxidant (Mufti \& Macchio 2009).

Flavonoids are water soluble plant pigments from fruits, vegetables, nuts and seeds besides being found in ginkgo biloba, St. John's wort and pycnogenol. Wine and bilberry contain anthocyanidins while flavanones are found in citrus fruits and cherries contain sufficient catechins glycoside. The flavonoids glycosides are used in cosmetics as antioxidant and anti-inflammatory compounds. Flavonoid rutin, derived from buckwheat, protects the blood vessels from fragility and improves microcirculation at the sub epidermal level (Somerset Cosmetic Company; Mufti \& Macchio 2009). Similarly, arbutin flavonoids, derived from the leaves of the ericaceae and rosaceae are used as a natural whitening agent in cosmetics. Soy isoflavonese are active against skin cancer according to some studies. Grape seed extract, containing flavonoids antioxidant, is comparable to soy extract containing isoflavones. Profound influence of flavonoids in cosmetics has been due to substantial increase in blood circulation at the sub epidermal level and an improvement of intra-cellular membrane exchanges of micronutrients (Mufti $\&$ Macchio 2009). They also improve venous insufficiency and capillary fragility resulting in increased oxygen supply to the cells and overall improvement of skin's texture and appearance.

Proteins, amino acids and peptides are widely incorporated into hair and skin care products to provide excellent conditioning, moisturizing and filmogenic actions (Mufti
\& Macchio 2009; Somerset Cosmetic Company). However, proteins from animal sources are replaced with those of marine and vegetable origin in cosmetics. On skin and hair, the hydrolyzed protein molecule exhibits extraordinary water binding and texturizing properties. It also reduces the split ends of damaged hair and induces superior gloss. Hygroscopic amino acids are mostly used in skin care products for their water-binding properties, as well as their improved elasticity, silky feel and skin firming effects. Enzyme hydrolysates and amino acids are moisturizers that penetrate the stratum corneum, epidermis and some dermal layers. On the contrary, higher molecular weight proteins do not penetrate significantly into the epidermis and remain on the surface of stratum corneum providing skin tightening or smoothing action (Mufti \& Macchio 2009). The natural wheat protein, hydrolyzed wheat protein, whole oats, hydrolyzed wheat protein polysiloxane and polyvinylpyrrolidone copolymers are just a few of the higher proteins that are used in cosmetics for their beneficial actions (Mufti \& Macchio 2009; Somerset Cosmetic Company).

Lipids covalently link with carbohydrates to form glycolipids and proteins forming lipoproteins and are used as emollients, moisturizers, viscosity modifiers, body builders, binders, emulsifiers for pleasant feel and above all as formula stabilizers (Mufti \& Macchio 2009). There are five major classes of lipids - neutral lipids, phospholipids, sterols, waxes and glycolipids (Mufti \& Macchio 2009). Stearic, palmitic, myristic, oleic, linoleic and linolenic acids are common ingredients in modern cosmetics. Biomimetic phospholipid complexes derived from some vegetable oils offer unique features for topical delivery and skin conditioning effects.

Cholesterol is widely used as water in oil emulsifier holding substantial moisture on the skin. New avocado sterols having an $80 \%$ active component with lubricious emollient action on 
the skin are used in place of animal-derived lanolin. Squalene, a sterol precursor found in plants, is also used widely in cosmetics as an emollient (Mufti \& Macchio 2009). Beeswax and vegetable derivatives are frequently used in cosmetics while silicone modified waxes improve feel and stability considerably. Ceramides fatty acids replenish moisture due to their water-binding capacity and help making skin soft and supple (Mufti \& Macchio 2009; Somerset Cosmetic Company).

Plant exudates including naturally occurring balsams, gums, oleoresins and resins, though insoluble in water and soluble in alcohol, often soften under heat are frequently used in cosmetics as binders and formula stabilizers (Mufti \& Macchio 2009). Seaweeds and algae are rich natural sources of essential amino acids, polyphenol glycosides, minerals, water soluble vitamins and trace elements. In color cosmetics, some iron oxide pigments are replaced with ground green, red, blue, brown and yellow algae. All these materials have strong antioxidant action (Mufti \& Macchio 2009).

\section{Delivery Systems}

Pharmaceutical delivery systems consist of formulations and devices that deliver therapeutic agents to the desired body locations and/or provide timely release of therapeutic agents. Drug delivery systems combine polymer science, pharmaceutics, bioconjugate chemistry and molecular biology while aiming to control drug pharmaco-kinetics/dynamics, non-specific toxicity, immuno-genicity and biorecognition in the search for an improved efficacy (European Technology Platform on Nanomedicine, 2005). It is important to note that drug delivery and targeting systems minimize drug degradation and loss, prevent harmful side effects and increase the availability of the drug at disease sites. Drug carriers include micro and nanoparticles, micro and nano capsules, lipoproteins, liposomes, and micelles that are engineered to react to stimuli, slowly degrade being site specific. Targeting mechanisms could be either passive or active. For example, preferential accumulation of chemotherapeutic agents in solid tumours due to differences in vascularisation of the tumour tissues compared to healthy tissues is known as passive targeting whereas in active targeting chemical 'decoration' of drug carrier surface with molecules enabling them to be selectively attached to diseased cells.

Controlled drug release is equally important for therapeutic success and it could be sustained or pulsatile type. Sustained release may involve diffusion based drug release from polymer or by degradation over a period of time whereas pulsatile release mimics a process similar to insulin production in body. This is facilitated by using drug conjugated polymers that respond to specific stimuli like - exposure to light or other source of radiation, changes in $\mathrm{pH}$ or temperature.

Most of the medicines are currently administered parenterally, but there is a clear-cut preference for other routes like oral, pulmonary or transdermal. Crossing of existing biological barriers by such large molecules is a challenging task that requires better understanding of macromolecular transport. However, any success attained would profoundly change the way nanotechnology based drugs are put to use in future. The oral route would require the precise knowledge of nanoformulations crossing the gastrointestinal tract epithelium overcoming enzymatic and permeability barriers. Similarly, pulmonary delivery is another method that should focus on aerodynamic characteristics of delivery systems and their ability to deliver drugs with improved bioavailability. Non-invasive pulmonary delivery involves extremely large surface area for drug absorption, thin alveolar epithelium, permitting rapid absorption and absence of first-pass metabolism and thus needs special attention for developing nanostructured 
systems. Especially in case of central nervous system diseases, the targeted delivery capable of crossing blood-brain barrier should be examined more in detail. Rational and high throughput screening methods for technologies to transport molecular species through biological barriers and target one or many specific locations is considered as an important issue to study in future.

\section{Nano-Devices - Targeted Delivery}

Ongoing activities of developing devices like programmable nanoneedles, nanofluidic components and assemblies, nanobiosensors for detecting the presence of therapeutic agents in body fluid combined with targeted delivery are extremely useful and should continue in future. Delivery systems could also be a part of biomedical devices for implantation or surgical tools that could provide real benefits for the patient. In future, more emphasis will be (European Technology Platform on Nanomedicine 2005; Nanomedicine Nanotechnology for Health 2006) on sustainable and pulsatile biochemical releases; micro-electro-mechanical systems/nanoelectro-mechanical systems (MEMS/NEMS) devices for controlled temporal and sequential multiple drug releases; gels, patches and sensor based devices; implantable biochips/ microfluidic devices; pill on a chip based technology; polymersome and liposome smart carriers; physical stimuli sensitive carriers and nanodevices comprising of metabolite sensor combined feedback based release mechanisms.

Carriers in targeted deliveries provide mechanisms to improve effectiveness of the macromolecules. A successful carrier system demonstrates optimal loading and release properties, long shelf-life and low toxicity. Colloidal and micellar solutions, vesicle and liquid crystal dispersions and a variety of nanoparticulate dispersions consisting of particles of $10 \mathrm{~nm}-500 \mathrm{~nm}$ diameter show great promise as described in brief here (European
Technology Platform on Nanomedicine 2005; Nanomedicine - Nanotechnology for Health 2006).

Micelle is a spherical or rod shaped molecular aggregate in a solution consisting of 10-100 surface active molecules. Hydrophilic and hydrophobic parts orient according to their interaction with the surrounding solvent. Bioactive compounds are encapsulated in a core surrounded by a hydrophilic shell protecting the contents and transporting at concentrations exceeding their intrinsic water solubility. It is possible to select the outer shell composition such that it escapes detection by reticuloendothelial system avoiding early elimination from the blood circulation. Micellar size, shape and chemical conjugations are easy to control to improve not only their stability but even their movements to selectively target a broad range of disease cells.

Liposomes are closed lipid vesicles surrounding an aqueous interior, appropriate to encapsulate exogenous materials for their ultimate delivery into the cells. These vesicles have one or more number of lipid bi-layers wherein molecular compounds are encapsulated and solubilised. Incorporation of size selective channel proteins in the liposome membrane converts them into size specific filters permitting diffusion of small solutes like ions, nutrients and antibiotics. Molecular compounds encapsulated in nanosize liposome structures with channel proteins are completely protected from premature degradation. However, the molecular compounds diffuse through channels driven by the concentration gradient between interior and exterior of the nanostructure.

Dendrimers are branched species of nanometre size macromolecules comprising of a central core, branching and terminal functional groups. Core chemistry determines the solubilising properties of the cavity within dendrimer whereas external chemical groups decide overall solubility and chemical 
behaviour. It is also possible to attach linkers to the external dendrimer surface which enable them to bind to a specific site while its stability and protection from phagocytes is achieved by surface functionalizing them with entities like polyethylene glycol chains.

Amorphous and crystalline nanoparticles having spherical or capsule like shapes can adsorb and/or encapsulate molecular compounds thus protecting them against chemical and enzymatic reactions. In a nanocapsule, the molecular compounds are confined to a cavity surrounded by a polymeric membrane while in nanospheres the compound is uniformly dispersed in a matrix. In recent years, biodegradable polymeric nanoparticles are becoming more popular in controlled release of molecular compounds in targeting particular organs/tissues in various forms like DNA carriers in gene therapy and oral deliveries of proteins, peptides and genes. Hydrogels - a three-dimensional polymer network that swells without dissolving in water are used to regulate molecular compound release in reservoir based carriers in swelling controlled release devices. Hydrogels could be intelligent and stimuli sensitive systems capable of modulating compound release in response to changes in parameters like $\mathrm{pH}$, temperature, ionic strength, electric field, specific analyte concentration gradient.

Molecular imprinted polymer or plastic antibody is another nanostructure that is formed in presence of a molecule which is removed later on leaving complementary cavities behind. Such polymers show chemical affinity for the original molecule and this could be used in biosensors, synthesizing catalysts and molecular separations. The behaviour is similar to that of antibodies or enzymes. These polymeric molecules may be employed in realizing a number of delivery modes e.g. rate programmed delivery where compound diffusion follows some specific rate profile; activation mediated compound delivery where the release is activated by some physical, chemical or biochemical process and feedbackregulated delivery where the release is regulated by the concentration of a triggering agent that is activated by the compound concentration in the body. Chemical conjugation of peptides and proteins with synthetic polymers is an efficient way of improving the control of nanostructures prepared from synthetic polymers for targeted delivery. Chemical conjugations of synthetic polymers to peptides or proteins can be used to reduce toxicity, prevent immunogenic or antigenic side reactions besides enhancing blood circulation time and improving drug solubility. Synthetic polymers and peptide conjugates can act as antibodies to specific epitopes and prevent random distribution of bioactive compounds throughout the patient's body by active targeting. Functionalization of synthetic polymers and peptides from extracellular protein matrix is an efficient way to mediate cell adhesion. However, the ability of the cationic peptides to complex DNA and oligonucleotides offers possibility of developing non-viral vectors for gene delivery.

Subcutaneous injection of liquid formulation producing solid structures are attractive in situ implants for non-oral applications because they are less invasive and painful compared to conventional implants. These implants deliver molecular compounds locally or systemically over prolonged periods of time extending to several months. This may be used for minimizing side effects by providing constant compound profiles especially important for delivering proteins with narrow therapeutic indices. Such systems are relatively simple and cost effective to manufacture.

\section{Different Administration Routes}

The success or failure of using a drug is influenced by choosing the way it is administered and therefore a delivery route can be decided by considering patient acceptability, drug solubility, ability to target the disease locations 
and effectiveness in dealing with the specific disease (Nanomedicine - Nanotechnology for Health 2006; European Technology Platform on Nanomedicine 2005).

The most important drug delivery route is through the mouth. Though increasing number of protein and peptide-based drugs offer huge potential for most effective therapeutics but they do not easily cross mucosal surfaces and biological membranes and are easily degraded, prone to rapid clearance in the liver and other body tissues and require precise dosing. Presently, protein drugs are administered by injection but this route is less patient friendly and also poses problems of oscillating blood drug concentrations. Despite gastrointestinal tract-related problems in the stomach, enzymatic degradation throughout the gastrointestinal tract and bacterial fermentation in the colon, the peroral route is still the most sought after one as it offers maximum convenience, cost effectiveness and manufacturing cost reductions.

Parenteral deliveries comprising of intravenous, intramuscular or subcutaneous administrations are common in general patient care. Nanosize liposomes are administered intravenously these days. Nanoscale drug/ compound carriers show great promises for improving delivery through nasal and sublingual routes that avoid first-pass metabolism and for difficult access ocular, brain and articular cavities. Peptides and vaccines are delivered systemically using nasal route through drug macromolecule and nanoparticle conjugates. In addition, a colloidal drug carrier is better option for improving the ocular drug deliveries.

Pulmonary delivery is carried out via aerosols, metered dose inhaler, dry powder inhalers and nebulizers that may contain liposomes, micelles, nanoparticles and dendrimers. Pulmonary delivery research is driven by its potential for successful protein and peptide drug delivery, promise of an effective gene delivery and need for replacing chlorofluorocarbon propellants in inhalers. This delivery provides local targeting for the treatment of respiratory diseases. However, success of protein drug pulmonary delivery is reduced by proteases in the lung that lower bioavailability and by barrier between capillary blood and alveolar air.

Non-invasive transdermal drug delivery avoids gastrointestinal irritation, metabolism, variations in delivery rates and interference due to the presence of food and is also suitable for unconscious patients. However, there are limitations of slow penetration rates, lack of dosage flexibility and/or precision and a restriction to relatively low dosage drugs.

Trans tissue local deliveries are fixed in resected tissues during surgery for giving better pharmacological effect and minimizing systemic and administration associated toxicities. This includes - drug/compound loaded gels that are formed in-situ and adhere to resected tissues releasing drugs/compounds, proteins or gene encoding adenoviruses; antibody fixed gels that form a barrier on a target tissue that could prevent cytokines permeation into that tissue; cell-based delivery that involves a gene transduced oral mucosal epithelial cellimplanted sheet; device directed delivery - a rechargeable drug infusion device that can be attached to the resected sites.

In gene delivery, a challenging task in treatment of genetic disorders, plasmid DNA is introduced into the target cells. It needs to be transcribed and the genetic information ultimately translated into the corresponding protein. The gene delivery system needs to target the desired cells, transported through the cell membrane, taken up and degraded in the endolysosomes and plasmid DNA intracellularly transported to the nucleus. 


\section{Delivery and Multitasking Medicines}

Delivery consists of nanosize elements for carrying the therapeutic and biologically active compound to the desired locations. In this context, nanotechnology not only offers possibility of pharmaceuticals deliveries or other therapeutic agents, but also utilities for diagnostics and regenerative medicine (European Technology Platform on Nanomedicine 2005; Nanomedicine Nanotechnology for Health 2006). For example, larger therapeutic systems like nanoherbals provide far more scope for diversity and complexity that makes their understanding still more challenging and their delivery more difficult. However, these larger systems do possess unique power to take care of more number of diseases such as in case of natural medicines. Such a combination of diagnosis, targeted delivery and regenerative medicine systems would therefore be ultimately capable of handling multiple tasks and even combine functions like diagnosis and therapy together leading to a completely new paradigm of human health care. Nanoherbals could be better choice in this context as compared to simple molecular compounds of modern medicine.

Delivery systems combining a much wider range of physical and chemical functional properties of nanomaterials, components and devices together are required to be developed in the near future for general (European Technology Platform on Nanomedicine 2005; Nanomedicine - Nanotechnology for Health 2006). List of such materials are components and devices would involve - liposomes, micelles and micro-emulsions; liquid and nanocrystals; antibodies and biochemical conjugates; natural proteins; polymer and bioconjugates; biodegradable nanoparticles and nanocapsules; virus like gene delivery system; nucleic acid or mimetic deliveries; vaccine delivery and synthetic biomimetic devices and systems. These entities should preferably be derived from herbal sources as well. Novel developments in these areas should focus on measuring efficacy and safety by conducting in vitro and in vivo experiments that may include the following aspects related to namely — polymersomes; prodrug therapy; smart and stimuli responsive drugs; in vivo imaging; systems localising and imaging agents and self-assembly based systems. Drug metabolism, pharmaco-kinetic and toxicological properties of such comprehensive systems of compounds, materials and devices should be carried out in detail using the methods approved by statutory bodies before they are put to clinical applications.

The process of recognising disease-specific molecules that are located either in the target cell membrane or in specific compartments within the cell is of very critical significance and importance in exercise. Any effort to enhance this function and reduce production costs is always welcome. The identification of such molecules can be carried out by using rational design, high throughput screening and even a combining the two. The important research (European Technology Platform on Nanomedicine 2005; Nanomedicine Nanotechnology for Health 2006) areas in this context could be related to the development of newer targeting agents; intracellular specific compartment targeting and multiple target approaches.

Proper delivery will be possible only in case there is deeper understanding of the interactions of the involved nanostructures within the human body. This can be facilitated by conducting in-vitro, ex vivo and in vivo experiments. Very little information is available in public domain on how these nanomedicines are transported and eliminated in vivo, and what the serious consequences are such as immunogenicity. Important areas (European Technology Platform on Nanomedicine 2005; Nanomedicine - 
Nanotechnology for Health 2006) to be considered would certainly include fundamental studies of interaction of nanostructures with plasma proteins and relation between protein adsorption and removal of nanostructures; cell absorption of nanostructures; nanostructure cell uptake and recycling; nanostructures transendocytosis; nanostructures endosomal escapes; nanostructure safety evaluation; in vivo carrier biodistribution and degradation and smart and intelligent nanostructures for biomedical applications.

\section{R\&D Priorities - Drug/Compound Delivery Development Programme}

Nanoparticles have already been used in drug delivery systems with success but nanoparticulate delivery systems especially involving nanoherbals have still greater potentials for many newer applications including anti-tumour, gene, AIDS and radio therapy, protein delivery, antibiotics, virostatics, vaccines and as vesicles to cross over the bloodbrain barrier (European Technology Platform on Nanomedicine 2005). Nanoparticles offer advantages regarding targeting, delivery and release and with their additional potential to combine diagnosis and therapy. These techniques are emerging as a major tool in multitasking nanomedicines (European Technology Platform on Nanomedicine 2005). The main hurdles faced are related to improving their stability in the biological environment, influencing the bio-distribution of active compounds, improving drug/compound loading, targeting, transport, release and interaction with existing biological barriers. Nanoparticle cytotoxicity and their degradation products are still not very well known and further improvements in biocompatibility are of major concern in future.

Some efforts were made in converting a number of herbal extracts into nanoforms using water soluble encapsulating shells (Ahmad et al. 2008; Islam \& Ahmad 2008). When applied to cell lines and animal disease models in rats there was clear evidence of significant dose reduction associated with almost complete cure of the diseases like liver cirrhosis and brain cell damage due to ischemia. Detailed histopathological tests carried out on trial animal organs (Islam \& Ahmad 2008) confirmed the changes in concentrations of the involved bio molecules taking place during treatment in the affected organs involved. This observation compares very well with those in case of traditional Chinese medicine tested elsewhere (Kang 2008) for treating liver fibrosis and cirrhosis in rat models following herbogenomic approach.

Keeping in view the existing competence level in $R \& D$ in contemporary nanomedicines (Nanomedicine - Nanotechnology for Health 2006; European Technology Platform on Nanomedicine 2005) future developments may involve research activities in areas such as delivery of large and highly localized quantities of drugs/compounds; release profile controls; development of better biocompatible, biodegradable and nontoxic nanomaterials; biomimetic polymers and nanotubes based architecture; molecular self-assembly; search for newer functions like - active targeting, on command delivery, intelligent release / bio-responsive trigger devices, self-regulated smart deliveries; intracellular delivery through virus like structures; development of Improved nanoparticle based implants; MEMS/NEMS based nanoparticle and multi reservoir release systems; nanoparticles based tissue engineering consisting of cytokines delivery for controlling cellular growth, differentiation and stimulate regeneration; biodegradable layered implant coatings and polymeric therapeutic peptide and proteins carriers to name specifically in this context.

\section{Nanoherbal Development Roadmap - A Proposal}

R\&D activities in the area of molecular medicines and related technologies have been going on for more than hundred years and 
with the advent of nanoscience and technology the pace has been accelerated further with better understanding of the related processes involved at atomic and molecular levels. Intelligent and smart nanomaterials synthesis is currently being attempted using organic, inorganic and biological macromolecules and their chemical conjugates, to handle various problems related to the development of nanomedicines as mentioned above (Nanomedicine - Nanotechnology for Health 2006). Having acquired enough expertise and advanced capabilities in this context world wide, in past few decades in the area of nanomedicines and related technologies, it has been possible to foresee the possible growth of R\&D and then predict some kind of roadmap on the same lines as it has been described in an elegant document prepared as European Technology Platform, Strategic Research Agenda for Nanomedicines, November 2006 (Nanomedicine - Nanotechnology for Health 2006).

Considering natural medicines derived out of herbal species as a rich source of phytochemicals in abundance, it is quite logical to consider some kind of development plans for nanoherbal medicines as a collection of pharmaceuticals, nutraceuticals and nutricosmetics running almost on the similar lines as it is seen in the case of molecular medicines. Of course, the situation in herbal medicines is far more complex than simple molecular structures involved in modern medicines and therefore the pace of progress in case of nanoherbal medicines is certainly going to take more time to mature for their clinical applications as compared to those of molecular medicines highlighted in the above referred roadmap. But, on the other hand, the rigors of the animal and human trial experiments are expected to be rather simplified as most of the ingredients are generally approved by FDA in form of food supplements. For qualifying as a standard pharmaceutical, nutraceutical and nutricosmetic compound, the related product development must go through the standard route of standardization, quality control and good manufacturing practices. All other advantages of the enabling technologies developed in connection with nanomedicines are expected to be applicable with nanoherbal medicines once applied with proper care. For example, in targeted delivery the carrier itself may be chosen as one of the herbal nanoforms instead of using much simpler nanostructured material with an eye on multitasking already pointed out as the next stage development of targeted deliveries of nanomedicines. Similarly, though far more complex but better understanding of nanostructured material interaction with human body and its organs will certainly enable us the use of nanoherbals to go through their in vitro and in vivo studies for various clinical trials at a later stage.

\section{TIME LINE OF DEVELOPMENT}

\section{Nanoherbal Pharmaceuticals}

- $1-2$ years

- Design of herbal pharmaceutical nanoparticles - nanoherbals

- Structural Stabilization - plant derived macromolecules

- Green Chemistry Synthesis - laboratory scale production - SFE

- $3-5$ years

- In vitro and in vivo barrier crossing abilities experiments

- Disease model animal experiments

- Surface functionalization of nanoherbals — targeted delivery applications

- Beyond 5 years

- Clinical trials involving models of cardiovascular diseases, cancer, musculoskeletal and inflammatory diseases, neurodegenerative diseases, diabetes and infectious diseases 


\section{Nanoherbal Nutraceuticals}

- 1 - 2 years

- Design of herbal nutraceutical nanoparticles - nanoherbals

- Structural Stabilization - plant derived macromolecules

- Green Chemistry Synthesis - laboratory scale production - SFE

- $3-5$ years

- In vitro and in vivo barrier crossing abilities experiments

- Disease model animal experiments

- Surface functionalization of nanoherbal nutraceuticals — targeted delivery applications

- Beyond 5 years

- Clinical trials for establishing anticholesterol, anti-oxidation, antiinflammation and bone protection processes.

\section{Nanoherbal Nutricosmetics}

- 1 - 2 years

- Design of herbal nutricosmetics nanoparticles - nanoherbals

- Structural Stabilization of plant derived macromolecules

- Green Chemistry Synthesis - laboratory scale production - SFE

- $3-5$ years

- In vitro and in vivo barrier crossing abilities experiments

- Animal model experiments

- Surface functionalization of nanoherbal nutricosmetics - targeted delivery applications
- Beyond 5 years

- Clinical trials of properties like antiaging, anti-wrinkle, anti-inflammatory skin diseases.

\section{Enabling Technologies}

- 1 - 2 years

- Nanoherbal particle synthesis - high pressure homogenization, nanoemulsification, nano-precipitation, SFE, hydrothermal and fluidized bed reactions

- In vitro models - transport through membranes and tissues. Better understanding of interaction of nanoherbals with human body and organs.

- Study of nanoherbals metabolism and pharmaco-kinetics

- Better delivery routes - oral, transdermal and pulmonary deliveries.

- Novel targeting entities involving natural herbal species.

- 3 - 5 years

- Blood-brain barrier crossing deliveries of nanoherbal species.

- Manufacturing cost reduction nanoherbal materials and devices.

- Immunogenicity prediction model.

\section{CONCLUSION}

Nanoherbals in the form of pharmaceuticals, nutraceuticals, food supplements and nutricosmetics have immense potential for their use in human healthcare related systems in future. Development of their targeted deliveries will not only reduce the consumptions of raw materials by several orders of magnitude but even enhance efficacies while reducing the unwanted side effects quite significantly. Using green chemistry-based processes like 
SFE and other for preparing nanoherbals will reduce the burden on environmental pollutions arising mainly due to discharge of effluents heavily loaded with a variety of organic and inorganic solvents. Phytochemical compounds derived from plants based nanocarriers, improved targeting molecules and further development of multitasking in targeted delivery; imaging and molecular level detection of molecular markers and other related functions will enhance the scope of their applications with additional advantages of biocompatible processes employed. Handling of chronic diseases as well as life style based ailments will possibly be better managed by combining the strength of molecular medicines with those of nanoherbals integrated in various forms proposed. Biotechnological developments to modify the genetic make up of individual herbs will certainly be used to get the right kind of phytochemical constituents without much dependence on environmental variations. Genetic engineering approach of minimizing the prevalent variations in phytochemicals in a given species will make the process of standardization more under control. The proposed roadmap for carrying out the research studies in coming times may be used as a guideline for planning activities in an interdisciplinary manner by involving expertises from different but related fields. Basic scientific studies supported by engineering and technological aspects of using the outcome of fundamental studies will be put to more effective uses in developing the useful applications possible in different fields. The chances of having significant contributions from the systematic pursuit of R\&D carried out in Malaysia involving a variety of herbs from the local rainforests is expected to be much brighter. It is sincerely hoped that commensurate support from the Government extended to the R\&D and academic institutions will catalyse the entire effort to pick up momentum in near future. International and National collaboration will be essential and beneficial to fill in the gaps in the comprehensive plan of advanced studies envisaged as very briefly highlighted in the proposed roadmap in this work. Proper networking and sharing of new knowledge acquired by the participating teams of experts working together on a continued basis will be necessary to contribute positively in the important task of improving the situation regarding availability of ensuring affordable human health care on a global level.

\section{ACKNOWLEDGEMENTS}

The authors are extremely thankful to Prof Kamrudin Hussin, Vice-Chancellor, University of Malaysia Perlis, for his constant encouragement throughout. Prof Zul Azhar Zahid Jamal, Prof Sazali Yaacob and Dr Rusli Abd. Hamid are acknowledged for their kind support to the authors for conducting this kind of futuristic study in the University.

Date of submission: May 2012

Date of acceptance: June 2012

\section{REFERENCES}

Ahmad, S, Samim, M, Islam, F \& Jalees, F 2008, 'Development of herbal Nanomedicines in Jamia Hamdard', in First Intl. Conf. on Nanotechnology, King Abdul Aziz University, Jeddah, KSA, 14-18 June.

European Technology Platform on Nanomedicine, Nanotechnology for Health 2005, 'Vision paper and basis for a strategic research agenda for nanomedicine', September 2005, <http://www. cordis.lu/nanotechnology/nanomedicine.htm>.

Islam, F \& Ahmad, S 2008, unpublished report.

Kang, YJ 2008, 'Herbogenomics: from traditional chinese medicine to novel therapeutics minireview', Exp. Biol. Med., vol. 233; pp. 1059-1065.

Mufti, J \& Macchio, R 2009, 'R\&D in the cosmetic age', <http://www.happi.com/>.

Nanomedicine - Nanotechnology for Health 2006, 'European technology platform, strategic research agenda for nanomedicines', $<\mathrm{http}$ ://cordis.europa. $\mathrm{eu} /$ nanotechnology/nanomedicine.htm $>$.

Somerset Cosmetic Company, LLC, P.O. Box 3372 , Renton, WA 98056, <www.makingcosmetics. com>. 